\title{
Application of compact finite difference method for solving some type of fractional derivative equations
}

\author{
Mahboubeh Molavi-Arabshahi ${ }^{1}$, Zahra Saeidi ${ }^{2}$ \\ 1,2 School of Mathematics, Iran University of Science \& Technology, \\ Narmak, Tehran 16844 Iran
}

Received: February 1, 2021. Revised: August 5, 2021. Accepted: September 1, 2021. Published: September 6, 2021.

\begin{abstract}
In this paper, the compact finite difference scheme as unconditionally stable method is applied to some type of fractional derivative equation. We intend to solve with this scheme two kinds of a fractional derivative, first a fractional order system of Granwald-Letnikov type 1 for influenza and second fractional reaction sub diffusion equation. Also, we analyzed the stability of equilibrium points of this system. The convergence of the compact finite difference scheme in norm $\|_{2}$ are proved. Finally, various cases are used to test the numerical method. In comparison to other existing numerical methods, our results show that the scheme yields an accurate solution that is quick to compute.
\end{abstract}

Keywords- Fractional wave sub-diffusion equation, Fractional derivative, Compact finite difference method, influenza equation

\section{INTRODUCTION}

here is a growing interest in the study of fractional differential equations among engineering, physics, mathematics and other sciences, because the dynamics of the behavior of phenomena can be studied more accurately by differential equations of non-integer derivative order [34]. The reason for this is described that in this type of equations, more detailed issues are considered, such as the history of the behavior of the devices, that maybe this feature is ignored in the equations of integer order [18].

Fractional differential equations used in modeling of many different processes and systems. Some applications of partial differential equations can be found in mechanics, quantum mechanics, chemistry, physics, light scattering, nuclear physics, spectrometers, and quantum field theory [31].

Inspired by fractional calculus, differential equations with fractional derivatives to describe and model many physical phenomena of image processing, chemical analysis of fluid flow in a liquid, model economic analysis of stock prices in financial mathematics, electromagnetism and so on. Therefore, in modeling, the behavior of phenomena by using differential and integral calculus can be better described by the fractional derivative order with their actual behavior [3], [7]. Among the fractional derivatives, we can mention Riemann-Liouville derivative and Jumarie derivative [35], which Jumarie derivative is defined based on finite differences and in [17] it is shown that this type of derivative is a generalization for Riemann-Liouville fractional derivative.

Also modeling high-order physical problems that do not have a long history in engineering. For example, fractional equations are used in image processing to eliminate noise and stabilize image edges.

By using fractional order operators and rewriting partial fractional differential equations, we can achieve to the goals of applying heat and energy equations to images and obtained stable solutions.

Using these equations will provide the boundaries of the image with varying degrees of clarity and preserve the edges of the image clearly. Fractional calculus is one of the most important applications of calculus. generally, the exact solution of most fractional differential equations is not available, so development numerical methods are of special importance for solving these equations. In recent years, many numerical methods have been proposed to solve these equations. Such as eigen vectors expansion [38], Homotopy perturbation method [26], [1], variational iteration method [33], [37], Adomian decomposition method [32], Finite difference method [12], [20], collocation method [15], [29], Legendre collocation method [13], Tau spectral Chebyshev transmission method [36], Bernstein polynomial [5], [6], The Legendre wavelet, The and many other methods.

Many explicit and implicit numerical methods for solving differential equations with fractional derivatives have been suggested thus far, with stability, convergence, and accuracy investigated. Because these methods do not have high accuracy. It seems a natural way to find and implement highorder methods.

Such methods are known as compact finite difference, which is a generalization of PADE approximations. Models for the transfer process in the form of fractional diffusion in time and space have attracted particular interest and have been 
investigated by a number of researchers [8], [28], [43]. The calculus fractional is almost as old as the calculus integer. The calculus fractional is almost as old as the calculus integer, the subject of calculus fractional for more than 300 years, which has been studied by Hospital, Leibniz, Riemann, Liouville, Granwald Letnikov, and other scientists [14], [40], [41].

The formation of fractional calculus, like many branches of mathematics, has taken place in a natural way. Fractional calculus is created by expanding and integrating the concepts of calculus in the integer order. Numbers, which are the first concepts we deal with in calculus, have evolved throughout history according to human needs. Early humans used numbers to count, so they only needed natural numbers. Over time, there was a need to define zero numbers and rational numbers, and then the need for continuity, for which real numbers were defined. Simple concepts have been generalized over time to meet existing needs, creating more general and complex concepts. Due to the fact that in nature there were dimensions that could not be described with natural dimensions, they had to develop the concept of non-integer numbers. Another concept that has been generalized over time is the factorial function, for which Euler found an integral expression for the factorial, which Legendre eventually defined the gamma function as a generalization of the factorial function to the realm of integers.

Most theories of fractional calculus were developed before the twentieth century. At first, mathematicians extended derivatives and integrals only to the fractional order and called it fractional calculus. When the derivative and integral were generalized to the desired order, the name remained, which is somewhat misleading.

Fractional differential calculus is a field of mathematical studies derived from the basic definitions of derivative and integral operators of ordinary calculus. The concept of fractional differential calculus is not new, the derivative and the integral of the fraction have been considered by mathematicians as a generalization of the derivative and integral of the integer order for a very long time.

Most of these popular definitions that are common in the world include Riemann-Liouville and Grunwald-Letnikov. Caputo also rewrote a number of classical definitions of Riemann-Liouville derivatives to solve fractional differential equations.

However, over the past few decades, fractional calculus has gained considerable importance and necessity in a wide range of applications in fields including engineering, chemistry, finance, physics, and so on. Many scientists believe that noninteger order derivatives are suitable for describing many phenomena in nature, and also provide useful tools for describing the persistence and hereditary properties of various processes and materials. In fact, it has been proven that models of fractional order relative to integer order models that have already been implemented are more suitable for describing the actual behavior of the system. Considering that obtaining the analytical solution of ordinary and partial differential equations of fractional order will not be easy in most cases, so by using appropriate numerical methods, the qualitative behavior of fractional order systems can be investigated.

In the early 1970s, for the first time, the compact finite difference method for approximating partial differential equations is applied to solve a number of fluid mechanical problems [4], [30], [42]. Since then, several separate classes of compact finite difference methods have been developed, two of the most common of which are central scheme. a major paper with an in-depth analysis of centralized compact schemes showed that these compact schemes for spectral waves have a spectral effect [27]. also Compact upwind schemes were developed to solve nonlinear hyperbolic problems. In recent years, compact schemes have been developed to more accurately and rapidly approximate partial differential equations. For example, compact schemes have been used soar several problems with wall-bounded currents same as the Navier-Stokes equations to simulate large opposite currents from the ultrasonic boundary layer current, as well as to scatter electromagnetic waves. Finite difference methods are used in solving the liquid-heat transfer equation or the power transfer, the Burger equation, and the wave equation.

Although compact finite difference methods using lower pore points can achieve higher order accuracy, they are implicit methods and usually require inverse matrix computation when used to solve partial differential equations. In an article [22], Lele examined compact finite difference methods in general and obtained their accuracy and efficiency. The methods proposed by Lele also include Kolatz. One of the most important advantages of the compact finite difference method is that the matrix of coefficients of the linear system of equations depends on the unknowns of the method used, which can be easily solved using the Thomas algorithm [2] [44].

With the spread of various sciences, including mathematics, attention to such calculations has increased. The different applications of fractional order equations in different disciplines have led many scientists to conduct research in this field.

Sun and $\mathrm{Wu}[39]$ investigated a finite difference method for a one-dimensional partial differential equation. Li et al. [23] proposed a numerical method in combine with a finite difference method in temporal direction and a finite element method in spatial direction for the one-dimensional spatial and temporal fraction wave equation. [19], $\mathrm{Hu}$ and Zhang proposed a finite difference method for the fourth-order fractional wave transfer equation, the discussing is on numerical methods for fractional wave equations. The most recent work on this subject is presented in the reference [11], [25], in which an implicit finite difference method is with first-order spatial accuracy.

The finite difference method is one of the differential methods that is widely used. Compared to other computational methods, the finite difference method is more efficient when the results are wide. A very important issue in this method is the spatial size of the model blocks and the number of frequencies is required. This determines the execution time of 
the program. The accuracy of the finite difference method increases to the desired extent with increasing the number of network blocks.

In recent years, the use of fractional order derivatives has provided a very useful tool to solve this type of problem. Fractional order derivatives are the general form of integer order derivatives that derivative order can be any positive real number instead of an integer. One of the most essential aspects of fractional order derivatives is that they are non-local, unlike fractional order derivatives. This means that the fractional order derivative of a function at a point depends not only on the properties of the function also it depends on the properties of the function at that point. The existence of such a property has made fractional order derivatives known as a very powerful way to study phenomena whose properties depend on space and time by Hong et al., 2008.

Ding et al. (2010) eliminated the effects of scale on the properties of porous media by using fractional order derivatives. In this case, the properties of the porous medium, such as hydraulic conductivity, will be scale-independent and have a constant value. Benson et al. (2000), Wittcraft \& Mirscritt (2008), Schumer et al. (2001) have used fractional order derivatives in many disciplines such as hydrogeology, finance, physics and electrical engineering. Benson et al. presented a fractional order displacement-diffusion equation to simulate solute transport in inhomogeneous saturated porous media

To the best of our knowledge, the methods listed above include accurate solutions up to second order. The goal of this study is to develop a more efficient numerical approach for solving fractional derivative problems. This system can be evaluated utilizing fourth-order compact finite differences.

The following is the outline for the paper:

We discussed the principles and definitions of the fractional derivative order in section 2 for two types of equations: a Granwald-Letnikov-type fractional model for influenza and fractional diffusion. The compact finite difference approach for solving fractional derivative equations, which was discussed in Section 2, is introduced in Section 3. We provide numerical examples in Section 4 to demonstrate the efficacy of compact finite difference approaches for solving fractional models.

\section{INTRODUCTION OF FRACTIONAL DIFFUSION EQUATIONS}

The Riemann-Liouville fractional derivative definition was instrumental in the development of fractional derivative theory and its application in mathematics. It has been discovered that fractional derivatives of the Caputo type provide a better representation of viscoelasticity and solid mechanics.

Definition 1 [20]:

Suppose "-n $<\alpha \leq \mathrm{n}$," which is a natural number. RiemannLiouville derivative of fractional order $\alpha$ is defined as follows:

$$
{ }_{a}^{R L} D_{t}^{\alpha} f(\mathrm{t})=\frac{1}{\Gamma(\mathrm{n}-\alpha)} \frac{d^{n}}{d t^{n}} \int_{a}^{t}(\mathrm{t}-\tau){ }^{n-\alpha-1} f(\tau) \mathrm{d} \tau
$$

In 1967, Caputo published an article defining the fractional derivative. It is clear from Caputo's definition of the fractional derivative that the Riemann-Liouville fractional integral is used for this definition, but in comparison with the RiemannLiouville fractional derivative we find that the derivatives of the integer order and fractional order integrals are shifted.

\section{Definition 2 [34]:}

Suppose $\mathrm{n}$ is a natural number and $-\mathrm{n}<\alpha<\mathrm{n}$. In this case the fractional derivative in the Caputo method is defined as follows:

${ }_{a}^{C} D_{t}^{\alpha} f(\mathrm{t})=\frac{1}{\Gamma(\mathrm{n}-\alpha)} \int_{a}^{t} \frac{f^{(\mathrm{n})}(\tau)}{(\mathrm{t}-\tau)^{\alpha+1-n}} \mathrm{~d} \tau$

Which $\alpha>0$, is called the fractional derivative order.

\section{Definition 3 [34]:}

The Granwald-Letnikov derivative of the fractional order $\alpha$ of the function $\mathrm{f}$ at point $\mathrm{t}$ is defined as follows becomes:

$$
{ }_{a}^{G L} D_{t}^{\alpha} f(\mathrm{t})=\lim _{h \rightarrow 0} h^{-\alpha} \sum_{k=0}^{n}(-1)\left(\begin{array}{l}
\alpha \\
k
\end{array}\right) f(\mathrm{t}-\mathrm{kh})
$$

where in

$$
n=\left[\frac{t-a}{h}\right],\left(\begin{array}{l}
\alpha \\
k
\end{array}\right)=\frac{\Gamma(\alpha+1)}{\Gamma(\mathrm{k}+1) \Gamma(\alpha-\mathrm{k}+1)},\left(\begin{array}{l}
\alpha \\
0
\end{array}\right)=1
$$

Rewriting Granwald Letnikov with $w_{k}^{(\alpha)}=h^{-\alpha}(-1)^{k}\left(\begin{array}{l}\alpha \\ k\end{array}\right)$ derivatives of the fraction as follows:

$$
{ }_{a}^{G L} D_{t}^{\alpha} f(\mathrm{t})=\lim _{h \rightarrow 0} h^{-\alpha} \sum_{k=0}^{\left[\frac{t-a}{h}\right]} w_{k}^{(\alpha)} f(\mathrm{t}-\mathrm{kh})
$$

Where the coefficients of " $w_{k}^{(\alpha)}$," are called Granwald Letnikov.

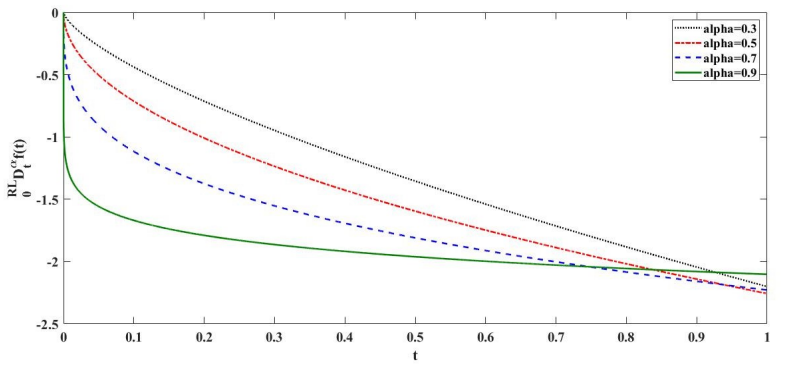

Fig. 1: Riemann-Liouville derivative operator of fractional order in different case $\alpha$

\section{A. . Fractional derivative equations system}

A typical assumption consistent for a broad class of dynamic systems in the mathematical description of physical processes is to consider the reliance of system behavior simply on current conditions, although there are systems whose behavior is insufficient to investigate and advance with this condition. It results in the system's poor performance while assessing its behavior. It is vital to obtain information about these systems' previous states in order to analyze their behavior. These systems are known as delayed systems.

Mathematical models have been used to investigate the transmission dynamics of influenza (flu). These models have been used to develop healthcare policies and forecast the 
effects of behavioral and biological interventions on disease outcomes. Sattenspiel and Herring (2003) developed a compartmental model to simulate influenza's regional spread and assess the efficacy of human quarantine. Using a compartmental model, Arino et al. $(2006,2008)$ explore the effects of antiviral treatment and/or vaccine on a developing pandemic using the parameters defined by Longini et al. (2004). As a result, the advantages of this simpler model that allows for sensitivity analysis in the situation of mixed approaches are demonstrated. Ferguson et al. 2005, modeled influenza transmission in Southeast Asia to look into the effects of mass antiviral medicine treatment as a control method.

In reference, Kasgrandi et al. [9] introduced a model for influenza in the general population, which consists of four groups (table 1).

Table 1: Variables at Population Level

\begin{tabular}{|c|c|}
\hline Variables & Description \\
\hline$\phi(\mathrm{t})$ & $\begin{array}{c}\text { number of people who are susceptible } \\
\text { Individuals at time } \mathrm{t}\end{array}$ \\
\hline$\psi(\mathrm{t})$ & number of people with the disease \\
\hline$\chi(\mathrm{t})$ & number of people who have improved \\
\hline$\kappa(\mathrm{t})$ & $\begin{array}{c}\text { the number of people which are safe } \\
\text { against disease }\end{array}$ \\
\hline
\end{tabular}

This model can be written as a first-order ordinary differential equation system as follows.

$$
\left\{\begin{array}{l}
\frac{d \phi(\mathrm{t})}{d t}=\mu(1-\phi(\mathrm{t}))-\beta \phi(\mathrm{t}) \psi(\mathrm{t})+\gamma \kappa(\mathrm{t}) \\
\frac{d \psi(\mathrm{t})}{d t}=\beta \phi(\mathrm{t}) \psi(\mathrm{t})+\sigma \beta \kappa(\mathrm{t}) \psi(\mathrm{t})-(\mu+\theta) \psi(\mathrm{t}) \\
\frac{d \chi(\mathrm{t})}{d t}=(1-\sigma) \beta \kappa(\mathrm{t}) \psi(\mathrm{t})+\theta \psi(\mathrm{t})-(\mu+\delta) \chi(\mathrm{t}) \\
\frac{d \kappa(\mathrm{t})}{d t}=\delta \chi(\mathrm{t})-\beta \kappa(\mathrm{t}) \psi(\mathrm{t})-(\mu+\gamma) \kappa(\mathrm{t})
\end{array}\right.
$$

where in is defined $\delta^{-1}$ reserved period (total safety), $\theta^{-1}$ contagious period, $\gamma^{-1}$ external safety course and $\beta$ the call rate. In order to improve the above integer order model, consider the fractional order model introduced by Kasgrandi in the reference [9].

$$
\left\{\begin{array}{l}
{ }_{a}^{G L} D_{t}^{\alpha} \phi(\mathrm{t})=\mu(1-\phi(\mathrm{t}))-\beta \phi(\mathrm{t}) \psi(\mathrm{t})+\gamma \kappa(\mathrm{t}) \\
{ }_{a}^{G L} D_{t}^{\alpha} \psi(\mathrm{t})=\beta \phi(\mathrm{t}) \psi(\mathrm{t})+\sigma \beta \kappa(\mathrm{t}) \psi(\mathrm{t})-(\mu+\theta) \psi(\mathrm{t}) \\
{ }_{a}^{G L} D_{t}^{\alpha} \chi(\mathrm{t})=(1-\sigma) \beta \kappa(\mathrm{t}) \psi(\mathrm{t})+\theta \psi(\mathrm{t})-(\mu+\delta) \chi(\mathrm{t}) \\
{ }_{a}^{G L} D_{t}^{\alpha} \kappa(\mathrm{t})=\delta \chi(\mathrm{t})-\beta \kappa(\mathrm{t}) \psi(\mathrm{t})-(\mu+\gamma) \kappa(\mathrm{t})
\end{array}\right.
$$

where in $\alpha \in[0,1]$. In this model, all defined parameters are assumed to be non-negative.

\section{B. Fractional diffusion equation}

The numerical solution to a fractional diffusion system subjected to a non-homogeneous field is the subject of this section. A fractional reaction sub diffusion equation is a linear integro-partial differential equation formed by replacing the first or second-order time derivative with a fractional derivative of order $\alpha>0$, in the classical diffusion or wave equation [34].

The fractional diffusion equation is another differential equation with fractional derivatives derived from the ordinary diffusion equation [10], [24].

The following fractional diffusion equation is investigated using a high-order compact finite difference technique.

In this section, the fractional diffusion equation is introduced. Then, using the Granwald-Letnikov approximation for the fractional derivative and the fourth-order approximation for the second derivative, a compact finite difference scheme is extracted and the stability analysis of this scheme is investigated. Consider the following fractional reaction-sub diffusion equation [23]:

$$
\begin{aligned}
& \frac{\partial u(\mathrm{x}, \mathrm{t})}{\partial t}={ }_{0}^{R L} D_{t}^{1-\alpha}\left[\mathrm{k}_{\alpha} \frac{\partial^{2} u(\mathrm{x}, \mathrm{t})}{\partial x^{2}}-\mathrm{k} u(\mathrm{x}, \mathrm{t})\right]+\mathrm{f}(\mathrm{x}, \mathrm{t}),{ }_{(3)} \\
& 0<\mathrm{x}<\mathrm{L}, 0<\mathrm{t} \leq \mathrm{T},
\end{aligned}
$$

In which the initial and boundary conditions are considered as follows:

$$
\begin{aligned}
& u(\mathrm{x}, 0)=\phi(\mathrm{x}), 0 \leq \mathrm{x} \leq \mathrm{L}, \\
& u(0, \mathrm{t})=\varphi(\mathrm{t}), \mathrm{u}(\mathrm{L}, \mathrm{t})=\psi(\mathrm{t}), 0<\mathrm{x} \leq T,
\end{aligned}
$$

Also, the functions $\phi(\mathrm{x}), \varphi(\mathrm{t}), \psi(\mathrm{t})$, known functions and $\mathrm{k}_{\alpha}, \mathrm{k}$, are assumed to be positive real constants. The fraction derivative of the Riemann-Liouville of the order $1-\alpha$, is introduced for function $u(\mathrm{x}, \mathrm{t})$, For numerical approximation Eq. (3) we define the spatial step length $h$ and the temporal step length $\tau$, as follows:

$$
\begin{aligned}
& n=0,1, \ldots, N \tau=\frac{T}{N}, t_{n}=n \tau, \\
& h=\frac{L}{M}, x_{i}=i h, i=0,1, \ldots, M .
\end{aligned}
$$

\section{COMPACT FINITE DIFFERENCE SCHEME}

In a considerable portion of numerical analysis, the compact finite difference strategy is employed as a basic method. The key argument is that it is simple to implement.

The fractional diffusion equation is another differential equation with fractional derivatives derived from the ordinary diffusion equation [14], [24]. This equation was first proposed by Nigmanalin in 1984. Numerous numerical methods have been proposed to solve this equation. [11] They did not have a high degree of accuracy, so the compact finite difference method was first used by Koi in 2009 to solve this equation. [14] From Chen's idea [23] and the method of intensive finite difference, they improved the accuracy of time

\section{A. fractional model for influenza using compact finite difference method}

Consider the following nonlinear differential equations 
$\frac{d Y(\mathrm{t})}{d t}=F(\mathrm{Y}(\mathrm{t})), \mathrm{Y}\left(\mathrm{t}_{0}\right)=Y_{0}, t \in\left[\mathrm{t}_{0}, \mathrm{~T}\right](4)$

Where vectors $\mathrm{F}, \mathrm{Y}_{0}, \mathrm{Y}(\mathrm{t})$ They are defined as follows.

$Y^{T}(\mathrm{t})=\left(\mathrm{y}_{1}(\mathrm{t}), \ldots, \mathrm{y}_{n}(\mathrm{t})\right)$,

$F(Y(\mathrm{t}))^{T}=\left(\mathrm{f}_{1}(\mathrm{y}), \ldots, \mathrm{f}_{n}(\mathrm{y})\right)$,

$Y_{0}^{T}=\left(\mathrm{y}_{1}(0), \mathrm{y}_{2}(0), \ldots, \mathrm{y}_{n}(0)\right)$.

Definition 4: A solution $\mathrm{Y}(\mathrm{t})$ from the device "Eq. (4)" is valid if it is positive whenever

$\mathrm{y}_{i}(0) \geq 0,1 \leq \mathrm{i} \leq \mathrm{n} \Rightarrow y_{i}(\mathrm{t}) \geq 0, \forall \mathrm{t}>0,1 \leq \mathrm{i} \leq \mathrm{n}$

According to the references [14], [21] it can be shown that the state variables $\mathrm{C}(\mathrm{t}), \mathrm{R}(\mathrm{t}), \mathrm{I}(\mathrm{t}), \mathrm{S}(\mathrm{t})$, from the system "Eq. (2)" is satisfied in positive condition

Definition 5: Suppose a typical first-order differential equation machine is given below.

$\frac{d Y(\mathrm{t})}{d t}=F(\mathrm{Y}(\mathrm{t})),(5)$

where in

$$
\begin{aligned}
& Y^{T}(\mathrm{t})=\left(\mathrm{y}_{1}(\mathrm{t}), \ldots, \mathrm{y}_{n}(\mathrm{t})\right), \\
& F(Y(\mathrm{t}))^{T}=\left(\mathrm{f}_{1}(\mathrm{y}), \ldots, \mathrm{f}_{n}(\mathrm{y})\right),
\end{aligned}
$$

We now define

$$
K(\mathrm{t})=\sum_{i=1}^{n} y_{\mathrm{i}}(\mathrm{t})
$$

If we set the variable $\mathrm{K}(\mathrm{t})$ in the first-order differential equation, the scalar value

$$
\frac{d K(\mathrm{t})}{d t}=f(\mathrm{~K}(\mathrm{t}))
$$

Where $f$ is a function dependent on the state variable $K(t)$, the so-called system "Eq. (5)" satisfied in conservation law.

Lemma 1: Set

$$
\Omega=\left\{(\phi, \psi, \chi, \kappa) \in R_{+}^{4}: \phi+\psi+\varphi+\kappa=1\right\} \text { relative to }
$$

the model "Eq. (2)" is positive persistent. To calculate the equilibrium points of the system "Eq. (2)" we put

$$
\begin{aligned}
& { }_{a}^{G L} D_{t}^{\alpha} \chi(\mathrm{t})=0 \quad{ }_{a}^{G L} D_{t}^{\alpha} \kappa(\mathrm{t})=0 \quad{ }_{a}^{G L} D_{t}^{\alpha} \psi(\mathrm{t})=0 \\
& { }_{a}^{G L} D_{t}^{\alpha} \phi(\mathrm{t})=0
\end{aligned}
$$

Therefore, the above system has equilibrium points $E_{\circ}=(0,1,1,1)$ and $E_{1}=\left(\phi_{1}, \psi_{1}, \chi_{1}, \kappa_{1}\right)$ where $\phi_{1}, \chi_{1}, \kappa_{1}$ are defined as follows.

$$
\begin{aligned}
& \phi_{1}=\frac{\mu+\theta}{\beta}-\sigma\left(\frac{\delta \theta \psi_{1}}{(\mu+\sigma \delta) \beta \psi_{1}+(\mu+\delta)(\mu+\gamma)}\right) \\
& \chi_{1}=\left(\frac{\theta \psi_{1}\left(\beta \psi_{1}+\mu+\gamma\right)}{(\mu+\sigma \delta) \beta \psi_{1}+(\mu+\delta)(\mu+\gamma)}\right) \\
& \kappa_{1}=\left(\frac{\theta \delta \psi_{1}}{(\mu+\sigma \delta) \beta \psi_{1}+(\mu+\delta)(\mu+\gamma)}\right)
\end{aligned}
$$

Where $\psi_{1}$, the positive root of the equation $g(\psi)=B_{1} \psi^{2}+B_{2} \psi+B_{3}\left(1-\chi_{0}\right)=0$,
Where the coefficients $B_{1}, B_{2}, B_{3}$, and the parameter $\chi_{0}$ defined as follows:

$B_{1}=\beta \mu(\theta+\mu+\delta \sigma)$,

$B_{2}=\beta \mu(-\beta \mu+\gamma(\delta+\theta+\mu)$

$+(\theta+\mu)(\delta+2 \mu)+\delta(-\beta+\mu)) \sigma$,

$B_{3}=\mu(\mu+\gamma)(\mu+\delta)(\mu+\theta)$

$\chi_{0}=\frac{\beta}{(\mu+\theta)}$

For the stability of the fractional order system "Eq. (2)" Suppose:

$f(\phi, \psi, \chi, \kappa)=\mu(1-\phi)-\beta \phi \psi+\gamma \kappa$

$\mathrm{g}(\phi, \psi, \chi, \kappa)=\beta \phi \psi+\sigma \beta \kappa \psi-(\mu+\theta) \psi$,

$\mathrm{h}(\phi, \psi, \chi, \kappa)=(1-\sigma) \beta \kappa \psi+\theta \psi-(\mu+\delta) \chi$,

$\mathrm{k}(\phi, \psi, \varphi, \kappa)=\delta \chi-\beta \kappa \psi-(\mu+\gamma) \kappa$

In this case, the Jacobin matrix of the above system corresponding to the equilibrium point $\mathrm{E}(\phi, \psi, \chi, \kappa)$ is obtained as follows.

$J(\mathrm{E})=\left(\begin{array}{llll}f_{\phi} & f_{\psi} & f_{\chi} & f_{\kappa} \\ g_{\phi} & g_{\psi} & g_{\chi} & g_{\kappa} \\ h_{\phi} & h_{\psi} & h_{\chi} & h_{\kappa} \\ k_{\phi} & k_{\psi} & k_{\chi} & k_{\kappa}\end{array}\right)$

Therefore

$$
J(1,0,0,0)=\left(\begin{array}{cccc}
-\mu & -\beta & 0 & \gamma \\
0 & \beta-\mu-\theta & 0 & 0 \\
0 & \theta & -(\mu+\delta) & 0 \\
0 & 0 & \delta & -\mu-\gamma
\end{array}\right)
$$

Where the Jacobian matrix elements are calculated at the equilibrium point " $E_{0}$ "

Theorem1: If $\chi_{0}=\frac{\beta}{(\mu+\theta)}<1$ then the equilibrium point $E_{0}$, for the system "Eq. (2)" is an asymptotically stable point and an unstable point for $\chi_{0}>1$.

In order to investigate the second equilibrium point, the Jacobi matrix corresponding to the equilibrium point $E_{1}$ is calculated as the following:

$$
J\left(\mathrm{E}_{1}\right)=\left(\begin{array}{cccc}
-\mu-\beta \psi_{1} & -\beta \phi_{1} & 0 & \gamma \\
\beta \psi_{1} & \beta \phi_{1}+\sigma \beta \kappa_{1}-(\mu+\theta) & 0 & \sigma \beta \psi_{1} \\
0 & (1-\sigma) \beta \kappa_{1}+\theta & -(\mu+\delta) & (1-\sigma) \beta \psi_{1} \\
0 & -\beta \kappa_{1} & \delta & -\beta \psi_{1}-(\mu+\gamma)
\end{array}\right)
$$

As a result, the characteristic equation corresponding to the equilibrium point $E_{1}$ is obtained as follows,

$(\lambda+\mu)\left(\lambda^{3}+b_{1} \lambda^{2}+b_{2} \lambda+b_{3}\right)=0$,

Where the coefficients $b_{1}, \mathrm{~b}_{2}, \mathrm{~b}_{3}$ are defined as follows.

$b_{1}=\gamma+\delta+2 \mu+2 \beta \psi_{1}$ 


$$
b_{2}=(\gamma+\mu)(\delta+\mu)+\beta \psi_{1}\left(\gamma+\delta+\theta+3 \mu+\sigma \delta+\beta \psi_{1}\right)-(\mu+\delta) \chi \approx-\mu \chi_{n+1}-\delta \chi_{n}
$$

$b_{3}=\frac{\left.\beta \psi_{1}(\psi(\delta+\mu)(\theta+\mu)+\gamma(\delta+\theta+\mu)-\delta \sigma \theta)+\mathrm{Q}\right)}{\eta+\beta(\mu+\delta \sigma) \psi_{1}}$

Where the values $\mathrm{Q}$ and $\psi$ are obtained by the following equations

$$
\begin{aligned}
& Q=\beta(\theta+\mu+\sigma \delta) \psi_{1}\left(2 \eta+\beta(\mu+\sigma \delta) \psi_{1}\right) \\
& \eta=(\mu+\gamma)(\mu+\gamma)
\end{aligned}
$$

If $E_{1} \in R_{+}^{3}$ according to Ruth-Hurwitz, we have:

$$
\text { - If } \quad D(\phi)>0, \mathrm{a}_{1}>0, a_{3}>0, \mathrm{a}_{1} a_{2}-a_{3}>0
$$

Equilibrium point $E_{1}$ is then a locally stable asymptote.

- If $D(\phi)<0, \mathrm{a}_{1}>0, a_{2}>0, \mathrm{a}_{1} a_{2}=a_{3}, \alpha \in[0,1)$

equilibrium point $E_{1}$ is then a locally stable asymptote.

$$
\begin{aligned}
& \text {-If } D(\phi)<0, \mathrm{a}_{1}<0, a_{2}<0, \alpha>\frac{2}{3} \text { Then } \\
& \text { equilibrium point } E_{1} \text { is unstable. }
\end{aligned}
$$

the

To derive numerical solutions for the system, the compact finite difference approach might be utilized "Eq. (2)".

Therefore, the system is discretized as a Granwald-Letnikov fractional derivative [16], [21].

$$
\begin{aligned}
& \sum_{j=0}^{n+1} \lambda_{j}^{(\alpha)} \phi_{n+1-j}=\mu\left(1-\phi_{n+1}\right)-\beta \phi_{n+1} \psi_{n}+\gamma \kappa_{n}, \\
& \sum_{j=0}^{n+1} \lambda_{j}^{(\alpha)} \psi_{n+1-j}=\beta \phi_{n+1} \psi_{n}+\sigma \beta \kappa_{n+1} \psi_{n}-(\mu+\theta) \psi_{n+1}, \\
& \sum_{j=0}^{n+1} \lambda_{j}^{(\alpha)} \chi_{n+1-j}=(1-\sigma) \beta \kappa_{n+1} \psi_{n}+\theta \psi_{n+1}-\mu \chi_{n+1}-\delta \chi_{n},
\end{aligned}
$$

$$
\sum_{j=0}^{n+1} \lambda_{j}^{(\alpha)} \kappa_{n+1-j}=\delta \chi_{n}-\beta \kappa_{n+1} \psi_{n}-\mu \kappa_{n+1}-\gamma \kappa_{n}
$$

The following results are obtained by comparing the equations "Eq. (2)" and the differential system "Eq. (6)".

Linear and nonlinear expressions in the right hand of the first equation at the point $\mathrm{t}_{k}=k$ are discretized as follows:

$$
\mu(1-\phi) \approx \mu\left(1-\phi_{n+1}\right), \gamma \kappa \approx \gamma \kappa_{n},-\beta \phi \psi \approx-\beta \phi_{n+1} \psi_{n},
$$

Linear and nonlinear expressions on the right-hand side of the second equation at the point $\mathrm{t}_{k}=k$ are discretized as follows:

$$
\begin{aligned}
& \beta \phi \psi \approx \beta \phi_{n+1} \psi_{n}, \sigma \beta \psi \kappa \approx \sigma \beta \kappa_{n+1} \psi_{n} \\
&-(\mu+\theta) \psi \approx-(\mu+\theta) \psi_{n+1}
\end{aligned}
$$

Linear and nonlinear expressions to the right of the third equation at the point $\mathrm{t}_{k}=k$ are discretized as follows:

$$
(1-\sigma) \beta \kappa \psi \approx(1-\sigma) \beta \kappa_{n+1} \psi_{n}, \theta \psi=\theta \psi_{n+1}
$$

Linear and nonlinear expressions to the right of the fourth equation at the point $\mathrm{t}_{k}=k$ are discretized as follows:

$\delta \chi \approx \delta \chi_{n},-\beta \kappa \psi \approx-\beta \kappa_{n+1} \psi_{n}$,

$-(\mu+\gamma) \kappa \approx-\mu \kappa_{n+1}-\mu \kappa_{n}$

The Granwald-Letnikov approximate is also used to discretize the derivative to the left of the "Eq. (2)". Now by manipulating the differential equation machine "Eq. (6)" it can be rewritten as follows.

$\phi_{n+1}=\frac{\mu+\gamma \kappa_{n}-\sum_{j=1}^{n+1} \lambda_{j}^{(\alpha)} \phi_{n+1-j}}{\lambda_{0}^{(\alpha)}+\mu+\beta \psi_{n}}$

$\psi_{n+1}=\frac{\beta \phi_{n+1} \psi_{n}+\sigma \beta \kappa_{n+1} \psi_{n}-\sum_{j=1}^{n+1} \lambda_{j}^{(\alpha)} \psi_{n+1-j}}{\lambda_{0}^{(\alpha)}+\mu+\theta}$

$$
\begin{aligned}
\chi_{n+1}= & \frac{(1-\sigma) \beta \kappa_{n+1} \psi_{n}+\theta \psi_{n+1}-\delta \chi_{n}-\sum_{j=1}^{n+1} \lambda_{j}^{(\alpha)} \chi_{n+1-j}}{\lambda_{0}^{(\alpha)}+\mu} \\
\kappa_{n+1}= & \frac{\delta \chi_{n}-\gamma \kappa_{n}-\sum_{j=1}^{n+1} \lambda_{j}^{(\alpha)} \kappa_{n+1-j}}{\lambda_{0}^{(\alpha)}+\mu+\beta \psi_{n}}
\end{aligned}
$$

Where $w_{j}^{(\alpha)}$ are the Granwald-Letnikov coefficients which are calculated recursively as below:

$\lambda_{j}^{(\alpha)}=\left(1-\frac{1+\alpha}{j}\right) \lambda_{j-1}^{(\alpha)}, j=1,2, \ldots$

B. The compact finite difference method for the fractional reaction sub diffusion equation

Outside the central differential part

$\frac{1}{h^{2}} \delta_{x}^{2} v_{i}=\frac{1}{h^{2}}\left(\mathrm{v}_{i-1}-2 \mathrm{v}_{i}+v_{j+1}\right)=$

$\left(\frac{\partial^{2} v}{\partial x^{2}}\right)_{i}+\frac{1}{12}\left(\frac{\partial^{4} v}{\partial x^{4}}\right)_{i} h^{2}+O\left(\mathrm{~h}^{4}\right)$,

Second order approximation gives for $\frac{\partial^{2} v}{\partial x^{2}}$. Now consider the following equations in order to obtain a quadratic approximation for $\frac{\partial^{2} v}{\partial x^{2}}$

$\left(\frac{\partial^{2} v}{\partial x^{2}}\right)_{i}=\left[2 \mathrm{hsinh}^{-1} \frac{\delta_{x}}{2}\right]^{2} v_{i}$

$=\frac{1}{h^{2}}\left[\delta_{x}-\frac{1^{2}}{2^{2} 3 !} \delta_{x}^{3}+\frac{1^{2} 3^{2}}{2^{4} 5 !} \delta_{x}^{5}-\frac{1^{2} 3^{2} 5^{2}}{2^{6} 7 !} \delta_{x}^{7}+\ldots\right]^{2} v_{i}=$ , $\frac{1}{h^{2}}\left[\delta_{x}^{2}-\frac{1}{12} \delta_{x}^{4}+\frac{1}{90} \delta_{x}^{6}-\frac{1}{560} \delta_{x}^{8}+\ldots\right] v_{i}$ 
where in $\delta_{x} v_{i}=v_{i+\frac{1}{2}}-v_{i-\frac{1}{2}}$

Now using "Eq. (7)" and "Eq. (8)" we will have: $\frac{\delta_{x}^{2}}{h^{2}\left(1+\frac{1}{12} \delta_{x}^{2}\right)} u_{i}^{n}=\frac{1}{h^{2}}\left(\delta_{x}^{2}-\frac{1}{12} \delta_{x}^{4}+\frac{1}{144} \delta_{x}^{6}-\frac{1}{1728} \delta_{x}^{8}+\ldots\right) \begin{aligned} & \text { simplified as follows } \\ & {\left[1+\mu_{2}+\left(\frac{1+\mu_{2}}{12}-\mu_{1}\right) \delta_{x}^{2}\right] \mathrm{v}_{i}^{n}=\left(1+\frac{1}{12} \delta_{x}^{2}\right) \mathrm{v}_{i}^{n-1}}\end{aligned}$

$=\left.\frac{\partial^{2} v}{\partial x^{2}}\right|_{i} ^{n}-\frac{1}{240 h^{2}} \delta_{x}^{6} v_{i}^{n}+O\left(\mathrm{~h}^{6}\right)=$

$\left.\frac{\partial^{2} v}{\partial x^{2}}\right|_{i} ^{n}-\left.\frac{1}{240} \frac{\partial^{6} v}{\partial x^{6}}\right|_{i} ^{n} h^{4}+O\left(\mathrm{~h}^{6}\right)$

Thus, the fourth order approximation is obtained for $\frac{\partial^{2} v}{\partial x^{2}}$. The

Riemann-Liouville fraction derivation discretization can also be considered as follows

$R L D_{t}^{1-\gamma} f(\mathrm{t})=\frac{1}{\tau^{1-\gamma}} \sum_{n=0}^{\left[\frac{t}{\tau}\right]} w_{n}^{1-\gamma} f(\mathrm{t}-\mathrm{n} \tau)+\mathrm{O}(\mathrm{h})$,

where in $w_{n}^{1-\gamma}$, Productive function coefficients is $w(\mathrm{z}, \alpha)=\sum_{n=0}^{\infty} w_{n}^{(\alpha)} z^{n} . \quad$ For $\quad w(\mathrm{z}, \alpha)=(1-\mathrm{z})^{\alpha}$ these coefficients are as follows:

$n=1,2, \ldots . w_{0}^{(\alpha)}=1, w_{n}^{(\alpha)}(-1)^{n}\left(\begin{array}{l}\alpha \\ n\end{array}\right)$

Which can be obtained recursively below

$w_{0}^{\alpha}=1, w_{n}^{\alpha}=\left(1-\frac{\alpha+1}{n}\right) w_{n-1}^{\alpha}, n \geq 1$.

Assume for convenience

$\lambda_{l} \equiv w_{n}^{1-\gamma}, l=0, \ldots, n$

So, with the using equation "Eq. (10)" we have

${ }_{0}^{R L} D_{t}^{1-\gamma} v\left(\mathrm{x}_{i}, \mathrm{t}_{n}\right)=\tau^{\gamma-1} \sum_{l=0}^{n} \lambda_{l} v\left(\mathrm{x}_{i}, \mathrm{t}_{n-1}\right)+\mathrm{O}(\tau)$

Thus using "Eq. (7)" and "Eq. (11)" and the forward difference formula

$\left.\frac{\partial v}{\partial t}\right|_{i} ^{n}=\frac{v_{i}^{n}-v_{i}^{n-1}}{\tau}+O(\tau)$

The compact finite difference scheme for the problem "Eq. (3)" is obtained as follows.

$\frac{v_{i}^{n}-v_{i}^{n-1}}{\tau} k_{\gamma} \frac{\tau^{\gamma-1}}{h^{2}} \sum_{l=0}^{n} \lambda_{l} \frac{\delta_{x}^{2}}{1+\frac{1}{12} \delta_{x}^{2}} v_{i}^{n-1}$

$-k \tau^{\gamma-1} \sum_{l=0}^{n} \lambda_{l} v_{i}^{n-1}+f_{i}^{n}, i=1,2, \ldots, M-1, \mathrm{n}=1,2, \ldots, \mathrm{N}$

where in

$v_{i}^{0}=\varphi\left(\mathrm{x}_{i}\right), \mathrm{i}=0,1,2, \ldots, \mathrm{M}$

$v_{0}^{n}=\phi\left(\mathrm{t}_{n}\right), \mathrm{v}_{m}^{n}=\psi\left(\mathrm{t}_{n}\right), \mathrm{n}=0,1, \ldots, \mathrm{N}$
If the parties to the relationship "Eq. (12)" multiply in $\tau\left(1+\frac{1}{12} \delta_{x}^{2}\right)$. By considering $\lambda_{0}=1$, the scheme can be $+\mu_{1} \sum_{l=1}^{n} \lambda_{l} \delta_{x}^{2} \mathrm{v}_{i}^{n-1}-\mu_{2} \sum_{l=1}^{n} \lambda_{l}\left(1+\frac{1}{12} \delta_{x}^{2}\right) \mathrm{v}_{i}^{n-1}+\tau\left(1+\frac{1}{12} \delta_{x}^{2}\right) f_{i}^{n}$ $i=1,2, \ldots, M-1, \mathrm{n}=1,2, \ldots, \mathrm{N}$

where in

$\mu_{1}=k_{\gamma} \frac{\tau^{\gamma}}{h^{2}}, \mu_{2}=k \tau^{\gamma}$

The plan "Eq. (13)" for the first-time step is as follows $\left(\frac{1+\mu_{2}}{12}-\mu_{1}\right) \mathrm{u}_{j-1}^{1}+\left[\frac{5}{6}\left(1+\mu_{2}\right)+2 \mu_{1}\right] \mathrm{u}_{j}^{1}+\left(\frac{1+\mu_{2}}{12}-\mu_{1}\right) \mathrm{u}_{j+1}^{1}$ $=\left[\frac{1}{12}+\lambda_{1}\left(\mu_{1}-\frac{\mu_{2}}{12}\right)\right] \mathrm{u}_{j-1}^{0}+\left[\frac{5}{6}-\lambda_{1}\left(2 \mu_{1}+\frac{5 \mu_{2}}{6}\right)\right] \mathrm{u}_{j}^{0}$ $+\left[\frac{1}{12}+\lambda_{l}\left(\mu_{1}-\frac{\mu_{2}}{12}\right)\right] \mathrm{u}_{j+1}^{0}+\frac{\tau}{12}\left(\mathrm{f}_{j-1}^{1}+10 \mathrm{f}_{j}^{1}+\mathrm{f}_{j+1}^{1}\right)$

We also have $2 \leq \mathrm{k} \leq \mathrm{N}$ بر for the time step $\left(\frac{1+\mu_{2}}{12}-\mu_{1}\right) \mathrm{u}_{j-1}^{k}+\left[\frac{5}{6}\left(1+\mu_{2}\right)+2 \mu_{1}\right] \mathrm{u}_{j}^{k}+\left(\frac{1+\mu_{2}}{12}-\mu_{1}\right) \mathrm{u}_{j+1}^{k}$ $=\left[\frac{1}{12}+\lambda_{1}\left(\mu_{1}-\frac{\mu_{2}}{12}\right)\right] \mathrm{u}_{j-1}^{k-1}+\left[\frac{5}{6}-\lambda_{1}\left(2 \mu_{1}+\frac{5 \mu_{2}}{6}\right)\right] \mathrm{u}_{j}^{k-1}$ $+\left[\frac{1}{12}+\lambda_{l}\left(\mu_{1}-\frac{\mu_{2}}{12}\right)\right] \mathrm{u}_{j+1}^{k-1}$

$\left.+\sum_{l=2}^{k} \lambda_{l}\left[\left(\mu_{1}-\frac{\mu_{2}}{12}\right)\right] \mathrm{u}_{j-1}^{k-l}-\left(2 \mu_{1}+\frac{5 \mu_{2}}{6}\right)\right] \mathrm{u}_{j}^{k-l}$

$\left.+\left(\mu_{1}-\frac{\mu_{2}}{12}\right)\right] \mathrm{u}_{j+1}^{k-l}+\frac{\tau}{12}\left(\mathrm{f}_{j-1}^{k}+10 \mathrm{f}_{j}^{k}+\mathrm{f}_{j+1}^{k}\right)$

Now, considering the law $\sum_{l=p}^{q} v^{l}=0$ for $\mathrm{q}<\mathrm{p}$, we can "Eq.

(13)" and "Eq. (14)" Wrote as follows

$\left(\frac{1+\mu_{2}}{12}-\mu_{1}\right) \mathrm{u}_{j-1}^{k}+\left[\frac{5}{6}\left(1+\mu_{2}\right)+2 \mu_{1}\right] \mathrm{u}_{j}^{k}+\left(\frac{1+\mu_{2}}{12}-\mu_{1}\right) \mathrm{u}_{j+1}^{k}$

$=\left[\frac{1}{12}+\lambda_{1}\left(\mu_{1}-\frac{\mu_{2}}{12}\right)\right] \mathrm{u}_{j-1}^{k-1}+\left[\frac{5}{6}-\lambda_{1}\left(2 \mu_{1}+\frac{5 \mu_{2}}{6}\right)\right] \mathrm{u}_{j}^{k-1}$

$+\left[\frac{1}{12}+\lambda_{1}\left(\mu_{1}-\frac{\mu_{2}}{12}\right)\right] \mathrm{u}_{j+1}^{k-1}$

$+\sum_{l=0}^{k-2} \lambda_{k-l}\left[\left(\mu_{1}-\frac{\mu_{2}}{12}\right) \mathrm{u}_{j-1}^{l}-\left(2 \mu_{1}-\frac{5 \mu_{2}}{6}\right) \mathrm{u}_{j}^{l}\right]$

$\left.+\left(\mu_{1}-\frac{\mu_{2}}{12}\right) \mathrm{u}_{j+1}^{l}\right]+\frac{\tau}{12}\left(\mathrm{f}_{j-1}^{k}+10 \mathrm{f}_{j}^{k}+\mathrm{f}_{j+1}^{k}\right)$

$1 \leq j \leq M-1,1 \leq k \leq N(16)$ 
Therefore, the matrix form of the scheme "Eq. (16)" can be obtained as follows

$$
\begin{aligned}
& A U^{1}=B_{0} U^{0}+F^{1}, \\
& A U^{k}=\sum_{l=0}^{k-1} B_{l}^{k} U^{l}+F^{k}, k=2,3, \ldots, N
\end{aligned}
$$

Where the tridiagonal matrices $A, B_{0}, B_{l}^{k}$ for $0 \leq l \leq k-1$ are defined as follows

$$
A=\left(\begin{array}{ccc}
10\left(1+\mu_{2}\right)+24 \mu_{1} & 1+\mu_{2}-12 \mu_{1} & \\
1+\mu_{2}-12 \mu_{1} & 10\left(1+\mu_{2}\right)+24 \mu_{1} & 1+\mu_{2}-12 \mu_{1} \\
\ddots & \ddots & \ddots \\
1+\mu_{2}-12 \mu_{1} & 10\left(1+\mu_{2}\right)+24 \mu_{1} & 1+\mu_{2}-12 \mu_{1} \\
& 1+\mu_{2}-12 \mu_{1} & 10\left(1+\mu_{2}\right)+24 \mu_{1}
\end{array}\right)
$$

$$
B_{0}=\left(\begin{array}{ccc}
10-\lambda_{1} q & 1-\lambda_{1} p & \\
1-\lambda_{1} p & 10-\lambda_{1} q & 1-\lambda_{1} p \\
\ddots & \ddots & \ddots \\
1-\lambda_{1} p & 10-\lambda_{1} q & 1-\lambda_{1} p \\
& 1-\lambda_{1} p & 10-\lambda_{1} q
\end{array}\right)
$$

With

$$
q=24 \mu_{1}+10 \mu_{2}, p=\mu_{2}-12 \mu_{1}
$$

And

$$
B_{l}^{k}=\lambda_{k-l}\left(\begin{array}{ccc}
-24 \mu_{1}-10 \mu_{2} & 12 \mu_{1}-\mu_{2} & \\
12 \mu_{1}-\mu_{2} & -24 \mu_{1}-10 \mu_{2} & 12 \mu_{1}-\mu_{2} \\
\ddots & \ddots & \ddots \\
12 \mu_{1}-\mu_{2} & -24 \mu_{1}-10 \mu_{2} & 12 \mu_{1}-\mu_{2} \\
& 12 \mu_{1}-\mu_{2} & -24 \mu_{1}-10 \mu_{2}
\end{array}\right)
$$

And for here the vectors $F^{1}$ and $F^{k}$ in "Eq. (17)" are defined as follows

$$
F^{1}=\left(\begin{array}{c}
X_{1}-\left(1+\mu_{2}-12 \mu_{1}\right) \mathrm{u}_{0}^{1}+\tau\left(\mathrm{f}_{0}^{1}+10 \mathrm{f}_{1}^{1}+\mathrm{f}_{2}^{1}\right) \\
\tau\left(\mathrm{f}_{1}^{1}+10 \mathrm{f}_{2}^{1}+\mathrm{f}_{3}^{1}\right) \\
\vdots \\
\tau\left(\mathrm{f}_{M-3}^{1}+10 \mathrm{f}_{M-2}^{1}+\mathrm{f}_{M-1}^{1}\right) \\
X_{2}-\left(1+\mu_{2}-12 \mu_{1}\right) \mathrm{u}_{M}^{1}+\tau\left(\mathrm{f}_{M-2}^{1}+10 \mathrm{f}_{M-1}^{1}+\mathrm{f}_{M}^{1}\right)
\end{array}\right)
$$

where in

$$
\begin{aligned}
& X_{1}=\left[1-\lambda_{1}\left(\mu_{2}-12 \mu_{1}\right)\right] \mathrm{u}_{0}^{0}, \\
& X_{2}=\left[1-\lambda_{1}\left(\mu_{2}-12 \mu_{1}\right)\right] \mathrm{u}_{M}^{0},
\end{aligned}
$$

And

$$
F^{k}=\left(\begin{array}{c}
Y_{1}-\left(1+\mu_{2}-12 \mu_{1}\right) \mathrm{u}_{0}^{k}+\tau\left(\mathrm{f}_{0}^{k}+10 \mathrm{f}_{1}^{k}+\mathrm{f}_{2}^{k}\right) \\
\tau\left(\mathrm{f}_{1}^{k}+10 \mathrm{f}_{2}^{k}+\mathrm{f}_{3}^{k}\right) \\
\vdots \\
\tau\left(\mathrm{f}_{M-3}^{k}+10 \mathrm{f}_{M-2}^{k}+\mathrm{f}_{M-1}^{k}\right) \\
Y_{2}-\left(1+\mu_{2}-12 \mu_{1}\right) \mathrm{u}_{M}^{k}+\tau\left(\mathrm{f}_{M-2}^{k}+10 \mathrm{f}_{M-1}^{k}+\mathrm{f}_{M}^{k}\right)
\end{array}\right)
$$

where in

$$
\mathrm{Y}_{1}=\sum_{l=0}^{k-2} \lambda_{k-l}\left(12 \mu_{1}-\mu_{2}\right) \mathrm{u}_{0}^{l}+\left[1-\lambda_{1}\left(\mu_{2}-12 \mu_{1}\right)\right] \mathrm{u}_{0}^{k-1}
$$

$\mathrm{Y}_{2}=\sum_{l=0}^{k-2} \lambda_{k-l}\left(12 \mu_{1}-\mu_{2}\right) \mathrm{u}_{M}^{l}+\left[1-\lambda_{1}\left(\mu_{2}-12 \mu_{1}\right)\right] \mathrm{u}_{M}^{k-1}$

\section{The stability of the compact finite difference scheme}

The concept of stability is related to the initial conditions of the problem, in such a way that if there is perturbation in the initial conditions of the problem, The numerical solution obtained means that the solution generated from the perturbation case is different from the numerical solution in the same step, but small enough for the initial conditions not perturbed.

The stability of the compact differential scheme is now investigated using the Fourier method. Suppose $\rho_{i}^{n}$ is the rounded error at the point $(\mathrm{k} \tau, \mathrm{jh})$. According to the scheme "Eq. (13)", the truncation error equations can be obtained as follows.

$$
\begin{aligned}
& {\left[1+\mu_{2}+\left(\frac{1+\mu_{2}}{12}-\mu_{1}\right) \delta_{x}^{2}\right] \rho_{i}^{n}=} \\
& \left(1+\frac{1}{12} \delta_{x}^{2}\right) \rho_{i}^{n-1}+\mu_{1} \sum_{l=1}^{n} \lambda_{l} \delta_{x}^{2} \rho_{i}^{n-1} \\
& -\mu_{2} \sum_{l=1}^{n} \lambda_{l}\left(1+\frac{1}{12} \delta_{x}^{2}\right) \rho_{i}^{n-1}
\end{aligned}
$$

Wherein $i=1,2, \ldots, M-1, \mathrm{n}=1,2, \ldots, \mathrm{N}$ also

$\rho_{0}^{n}=\rho_{M}^{n}=0,1 \leq n \leq N(19)$

Now for $\rho^{n}=\left(\rho_{1}^{n}, \rho_{2}^{n}, \ldots, \rho_{M-1}^{n}\right)^{T}$ consider the following norm

$$
\left\|\rho^{n}\right\|_{l^{2}}=\left(\sum_{i=1}^{M-1} h\left|\rho_{i}^{n}\right|^{2}\right)^{\frac{1}{2}}
$$

Assume that the solutions to the equations "Eq. (18)" and "Eq. (19)" are given below.

$$
\rho_{i}^{n}=G_{k} e^{j \sigma i h}, 1 \leq i \leq M-1,1 \leq n \leq N
$$

where in

$$
\sigma=\frac{2 \pi m}{L}, \mathrm{~m} \in Z
$$

By inserting the phrase "Eq. (21)" in "Eq. (18)" the result is obtained as follows.

$$
\begin{aligned}
& {\left[1+\mu_{2}+\left(-\frac{1+\mu_{2}}{3}+4 \mu_{1}\right) \sin ^{2}\left(\frac{\sigma h}{2}\right)\right] \mathrm{G}_{n}} \\
& =\left[1-\lambda_{1} \mu_{2}+\left(-\frac{1-\lambda_{1} \mu_{2}}{3}-4 \lambda_{1} \mu_{1}\right) \sin ^{2}\left(\frac{\sigma h}{2}\right)\right] \mathrm{G}_{n-1} \\
& -\left[\mu_{2}+\left(-\frac{\mu_{2}}{3}+4 \mu_{1}\right) \sin ^{2}\left(\frac{\sigma h}{2}\right)\right] \sum_{l=0}^{n-2} \lambda_{n-1} \mathrm{G}_{n}, 1 \leq n \leq N
\end{aligned}
$$

as a result

$$
G_{1}=\frac{1-\lambda_{1} \mu_{2}+\left(-\frac{1-\lambda_{1} \mu_{2}}{3}-4 \lambda_{1} \mu_{1}\right) \sin ^{2}\left(\frac{\sigma h}{2}\right)}{1+\mu_{2}+\left(-\frac{1+\mu_{2}}{3}-4 \mu_{1}\right) \sin ^{2}\left(\frac{\sigma h}{2}\right)} G_{0},
$$




$$
\begin{gathered}
G_{n}=\frac{1-\lambda_{1} \mu_{2}+\left(-\frac{1-\lambda_{1} \mu_{2}}{3}-4 \lambda_{1} \mu_{1}\right) \sin ^{2}\left(\frac{\sigma h}{2}\right)}{1+\mu_{2}+\left(-\frac{1+\mu_{2}}{3}-4 \mu_{1}\right) \sin ^{2}\left(\frac{\sigma h}{2}\right)} G_{n-1} \\
-\frac{\mu_{2}+\left(-\frac{\mu_{2}}{3}+4 \mu_{1}\right) \sin ^{2}\left(\frac{\sigma h}{2}\right)}{1+\mu_{2}+\left(-\frac{1+\mu_{2}}{3}-4 \mu_{1}\right) \sin ^{2}\left(\frac{\sigma h}{2}\right)} \sum_{l=0}^{n=2} \lambda_{n-l} G_{n-1},
\end{gathered}
$$

$2 \leq n \leq N$

\section{Theorem 2:}

Implicit finite difference scheme defined in "Eq. (13)" for $0<\gamma<1$, so Unconditionally stable.

Proof:

Using "Eq. (20)" "Eq. (21)" and we also "Eq. (8)" have:

$\left\|\rho^{n}\right\|_{l^{2}}^{2}=h \sum_{i=1}^{M-1}\left|\mathrm{G}_{n} e^{j \sigma i h}\right|^{2}=h \sum_{i=1}^{M-1}\left|G_{n}\right|^{2} \leq h \sum_{i=1}^{M-1}\left|G_{0}\right|^{2}$

$=h \sum_{i=1}^{M-1}\left|G_{n}\right|^{2}=h \sum_{i=1}^{M-1}\left|G_{0} e^{j \sigma i h}\right|^{2}=\left\|\rho^{0}\right\|_{l^{2}}^{2}$

Therefore, with a negligible perturbation in the initial conditions, the solution is smaller at each time step, and therefore the scheme is unconditionally stable.

\section{Truncation error of the method}

Using the equations "Eq. (9)"and "Eq. (11)"and the forward difference formula for the first-order derivative, the Truncation error of the scheme (13) is obtained as follows.

$R_{j}^{n}=\frac{\mathrm{v}\left(\mathrm{x}_{i}, \mathrm{t}_{n}\right)-v\left(\mathrm{x}_{i}, \mathrm{t}_{n-1}\right)}{\tau}-$

$k_{\gamma} \frac{\tau^{\gamma-1}}{h^{2}} \sum_{k=0}^{n} \lambda_{k} \frac{\delta_{x}^{2}}{1+\frac{1}{12} \delta_{x}^{2}} v\left(\mathrm{x}_{i}, \mathrm{t}_{n-1}\right)$

$+k \tau^{\gamma-1} \sum_{k=0}^{n} \lambda_{k} v\left(\mathrm{x}_{i}, \mathrm{t}_{n-k}\right)-\mathrm{f}\left(\mathrm{x}_{i}, \mathrm{t}_{n}\right)=\frac{\partial}{\partial t} v\left(\mathrm{x}_{i}, \mathrm{t}_{n}\right)$

$-\mathrm{k} \tau^{\gamma-1} \sum_{k=0}^{n} \lambda_{k}\left(\frac{\partial^{2}}{\partial x^{2}} \mathrm{v}\left(\mathrm{x}_{i}, \mathrm{t}_{n-1}\right)-\frac{h^{4}}{240} \frac{\partial^{6}}{\partial x^{6}} \mathrm{v}\left(\mathrm{x}_{i}, \mathrm{t}_{n-1}\right)+\ldots\right)$

$+k \tau^{\gamma-1} \sum_{k=0}^{n} \lambda_{k} v\left(\mathrm{x}_{i}, \mathrm{t}_{n-1}\right)-\mathrm{f}\left(\mathrm{x}_{i}, \mathrm{t}_{n}\right)+\mathrm{O}(\tau)$

$=\frac{\partial}{\partial t} v\left(\mathrm{x}_{i}, \mathrm{t}_{n}\right)$

$-{ }_{0}^{R L} D_{t}^{1-\gamma}\left[\mathrm{k}_{\gamma} \frac{\partial^{2} v\left(\mathrm{x}_{i}, \mathrm{t}_{n}\right)}{\partial x^{2}}-\mathrm{kv}\left(\mathrm{x}_{i}, \mathrm{t}_{n}\right)\right]-\mathrm{f}\left(\mathrm{x}_{i}, \mathrm{t}_{n}\right)$

$+k_{\gamma}\left(\frac{h^{4}}{240}\right){ }_{0}^{R L} D_{t}^{1-\gamma} \frac{\partial^{6}}{\partial x^{6}} v\left(\mathrm{x}_{i}, \mathrm{t}_{n}\right)+\mathrm{O}(\tau)+\mathrm{O}\left(\mathrm{h}^{6}\right)$

\section{NUMERICAL EXPERIMENTS}

We intend to examine the influenza equation for the compact finite difference method in different modes.

\section{Example 1:}

The numerical results of the compact finite difference scheme presented for the rank system Deficit is expressed. To check the stability of the equilibrium point $E_{0}=(1, \circ, \circ, \circ)$ Suppose $\mu=0 / 02, \quad \theta=73, \quad \beta=40$, Therefore $\chi_{\circ}=0 / 547$ it will be obtained. As a result, the 4 equilibrium points $E_{0}$ are locally stable asymptotes.

Figure 3 shows that the system is asymptotically stable at $E_{0}=(1, \circ, \circ, \circ)$. Also, by choosing $\beta=200$, $\chi_{\circ}=2 / 738$ is obtained. Therefore, the 4 equilibrium points $E_{0}$ are unstable.

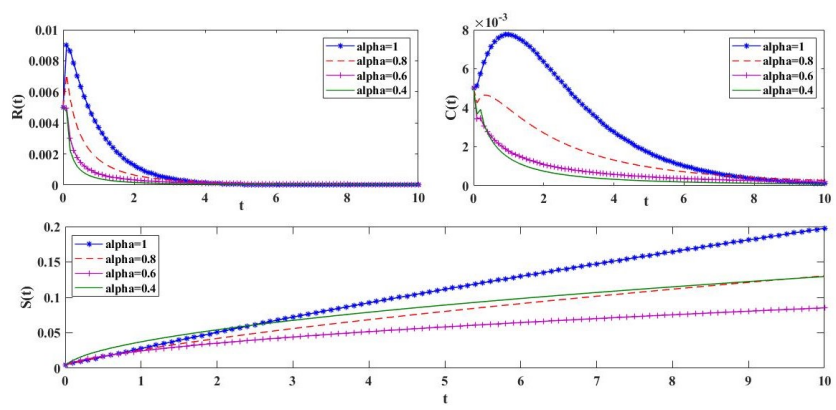

Fig. 3: The chart $\mathrm{R}(\mathrm{t}), \mathrm{S}(\mathrm{t})$ and $\mathrm{C}(\mathrm{t})$ for $\mu=0 / 02, \beta=40, \delta=1, \sigma=0 / 05, \theta=73, \gamma=0 / 5$

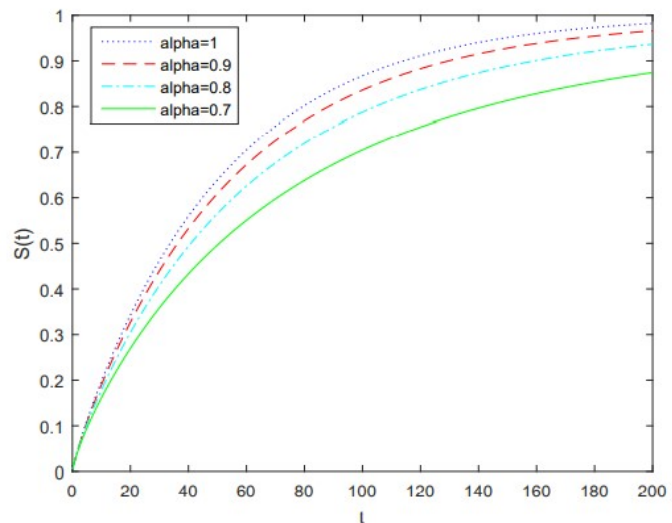

Fig. 4: the chart $\mathrm{S}(\mathrm{t})$ for different $\alpha$ and $\mu=0 / 02, \beta=40, \delta=1, \sigma=0 / 05, \theta=73, \gamma=0 / 5$

\section{Example 2.}

Consider the following a time-fraction sub-diffusion equation

$\frac{\partial^{\alpha} u(\mathrm{x}, \mathrm{t})}{\partial t^{\alpha}}=\frac{\partial^{2} u(\mathrm{x}, \mathrm{t})}{\partial x^{2}}+\frac{2 e^{x} t^{2-\alpha}}{\Gamma(3-\alpha)}-t^{2} e^{x}$,

with the initial and boundary condition:

$$
\begin{aligned}
& \mathrm{u}(\mathrm{x}, 0)=0,0<\mathrm{x}<1, \\
& \mathrm{u}(0, \mathrm{t})=\mathrm{t}^{2}, u(1, \mathrm{t})=\mathrm{et}^{2}, 0 \leq t \leq T
\end{aligned}
$$

The exact solution is given as follows.

$u(\mathrm{x}, \mathrm{t})=\mathrm{t}^{2} e^{x}$

We take $\alpha=0.5$ and $h, \tau=0.05$ 

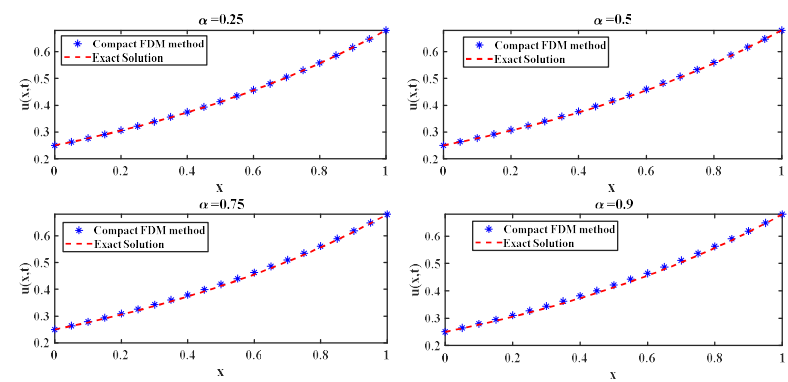

Fig 5: numerical and exact solution for

$$
\alpha=0.25,0.5,0.75,0.9 \text { and } h, \tau=0.05
$$

In Figure 5 is shown the numerical solution of the compact finite difference scheme.
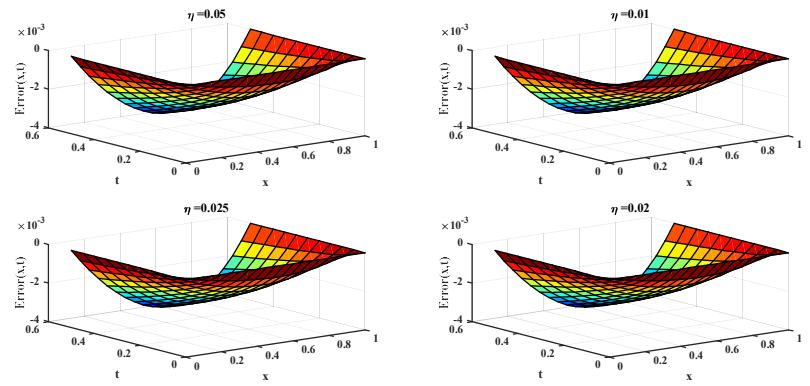

Fig 6: error for $\tau=0.05,0.01,0.025,0.02$

where $\alpha=0.75$ the temporal increment is small enough, the spatial convergence order is also shown in Table 1 . The numerical convergence order $O\left(h^{4}\right)$ in the spatial direction is, of necessity, confirmed.

In table 1 , we reported for different cases $\alpha$ of error and convergence order. Also, we verify estimated error for $\mathrm{T}=1$ is assumed as following relation:

$$
\|E\|_{2}=\left\|u_{\text {exact }}^{N}-u_{\text {Approximated }}^{N}\right\|_{2}
$$

The convergence order is assumed with relation

$$
o(h, \tau)=\log _{2}\left(\frac{\left\|E\left(h, \frac{\tau}{2}\right)\right\|_{2}}{\|E(h, \tau)\|_{2}}\right)
$$

Table 1: Error and convergence results in case $\alpha=0.5$

\begin{tabular}{|c|c|c|}
\hline$(h, \tau)$ & $\|E\|_{2}$ & $o(h, \tau)$ \\
\hline$\left(\frac{1}{2^{2}}, \frac{1}{2^{2}}\right)$ & $6.0308 \mathrm{e}-003$ & 2.0661 \\
\hline$\left(\frac{1}{2^{3}}, \frac{1}{2^{3}}\right)$ & $4.52777-003$ & 3.1958 \\
\hline$\left(\frac{1}{2^{3}}, \frac{1}{2^{6}}\right)$ & $3.0289 \mathrm{e}-003$ & 3.2363 \\
\hline$\left(\frac{1}{2^{4}}, \frac{1}{2^{7}}\right)$ & $4.8802 \mathrm{e}-004$ & 3.8377 \\
\hline
\end{tabular}


difference approach, and it was shown that these methods are unconditionally stable. The efficiency of finite difference methods and intensive finite difference methods is demonstrated numerically.

A compact difference approach is used to analyze a class of fractional sub-diffusion problems in this research. To solve the problem, we use a combination of compact approximation for discretizing the temporal variable and a fourth-order compact difference scheme for discretizing the spatial variable. In temporal and spatial variables, this type of compact difference method achieves (2-gamma) order and fourth-order accuracy, respectively. The scheme is more accurate than ones presented by many others. The energy approach is used to check the scheme's stability and convergence in maximal norm, where a new inner product is introduced. This inner product plays a critical role in our analysis of maximal norm estimates, which is one of the paper's primary contributions. We can also verify that the difference scheme (5.1) is convergent with the convergence order f $O\left(\tau^{\alpha}+h^{2}\right)$ of in maximum norm using the analytical method presented in this article.

\section{References}

[1] O. Abdulazi, I. Hashim and S. Momani, "Solving systems of fractional differential equations by homotopyperturbation method," Phys. Lett, A., 3722009, pp. 451459.

[2] E. Ahmed, A.M.A. El-Sayed and H.A. El-Saka, "On some Routh-Hurwitz conditions for fractional order differential equations and their applications in Lorenz, Rössler, Chua and Chen systems," Physics Letters A., 358(1) 2006, pp. $1-4$.

[3] O.P. Agrawal, "Response of a diffusion-wave system subjected to deterministic and stochastic fields," Z. Angew. Math. Mech. 83 (4) 2003, pp.265-274.

[4] W.A. Al-Salam, T.S. Chihara, "Convolutions of orthonormal polynomials," SIAM Journal on Mathematical Analysis, 7(1) 1976, pp.16-28.

[5] J. Arino, F. Brauer, P. van den Driessche, J. Watmough, and J. Wu, "Simple models for containment of a pandemic. Journal of the Royal Society," Interface, 3(8),2006, pp.453-457. https://doi.org/10.1098/rsif.0112.

[6] A. H. Bhrawy, M. M. Tharwat and A. Yildirim, "A new formula for fractional integrals of Chebyshev polynomials, application for solving mulit-term fractional differentials," J. Appl. Math. Model, 37 (6) 2013, pp. 4245-4252

[7] J. Cao, Y. Qiu and G. Song, "A compact finite difference scheme for variable order subdiffusion equation," Communications in Nonlinear Science and Numerical Simulation 48 2017, pp.140-149.

[8] M. Caputo and M. Fabrizio, "A new definition of fractional derivative without singular kernel, Progr. Fract. Differ," Appl, 1(2) 2015, pp.1-13.

[9] R. Casagrandi, L. Bolzoni, S.A. Levin and V. Andreasen, "The SIRC model and influenza A. Mathematical biosciences," 200(2) 2006, pp.152-169.

[10] S. Chen, F. Liu, P. Zhuang and V. Anh, "Finite difference approximations for the fractional Fokker-Planck equation," Applied Mathematical Modelling, 33(1) 2009, pp.256-273.

[11]M. Cui, "Compact exponential scheme for the time fractional convection-diffusion reaction equation with variable coefficients," Journal of Computational Physics, 280 2015, pp.143-163.

[12] V. Daftardar-Gejj and H. Jafari, "Solving a multi-order fractional differential equation using decomposition," Appl. Math. Comput, 1892007, pp.541-548.

[13]F. Dubois and S. Mengue, "Mixed collocation for fractional differential equations," Numer. Algor, 34 2003, pp.303-311.

[14]M. El-Shahed and A. Alsaedi, "The fractional SIRC model and influenza A," Mathematical problems in Engineering, 2011 (2011).

[15] N. Ferguson, D. Cummingsn and S. Cauchemez, et al., "Strategies for containing an emerging influenza pandemic in Southeast Asia," Nature 437. 2005, pp. 209214. https://doi.org/10.1038/nature04017.

[16] G.H. Gao, Z.Z. Sun, "A compact finite difference scheme for the fractional sub-diffusion equations," Journal of Computational Physics, 230(3) 2011, pp.586-595.

[17] G.C. González-Parra, M. Díaz-Rodriguez and V. Comezaquira, "A Nonstandard finite difference scheme for an Epidemic Model of Fractional Order," Avances En Simulación Computacional Y Modelado Numérico E. Dávila, G. Uzcátegui, M. Cerrolaza (Editores) (2012).

[18] N. Heymans, and I. Podlubny, "Physical interpretation of initial conditions for fractional differential equations with Riemann-Liouville fractional derivatives." Rheologica Acta, 45(5),2006, pp.765-771.

[19]X. Hu, L. Zhang, "On finite difference methods for fourth-order fractional diffusion-wave and sub diffusion systems," Applied Mathematics and Computation, 218(9),2012, pp. 5019-5034.

[20]A. Jajarmi, S. Arshad D. Baleanu, "A new fractional modelling and control strategy for the outbreak of dengue fever," Physica A, 535, 2019, pp. 122524.

[21] L. Jódar, R.J. Villanueva, A.J. Arenas and G.C. González, "Nonstandard numerical methods for a mathematical model for influenza disease," Mathematics and Computers in simulation, 79(3) 2008, pp .622-633.

[22] S.K. Lele, "Compact finite difference schemes with spectral-like resolution," Journal of computational physics, 103(1) 1992, pp.16-42.

[23]C.P. Li, Z.G. Zhao and Y.Q. Chen", Numerical approximation of nonlinear fractional differential equations with sub diffusion and super diffusion," Comput. Math. Appl. 62 2011, pp.855-875

[24] R. Lin and F. Liu", Fractional high order methods for the nonlinear fractional ordinary differential equation," Nonlinear Analysis: Theory, Methods \& Applications., 66(4)2007, pp.856-869.

[25]F. Liu, P. Zhuang and K. Burrage", Numerical methods and analysis for a class of fractional advection-dispersion models," Computers \& Mathematics with Applications, 64(10) 2012, pp. 2990-3007.

[26] Jr. Ira M. Longini, M. E. Halloran, A. Nizam and Y. Yang", Containing Pandemic Influenza with Antiviral Agents, American Journal of Epidemiology," Volume 
159, Issue 7, 1 April, Pages, 2004 pp.623633, https://doi.org/10.1093/aje/kwh092

[27] Y. Luchko, F. Mainardi, Cauchy and signaling problems for the time-fractional diffusion-wave equation," Journal of Vibration and Acoustics, 136(5) (2014).

[28] J.T. Machado, V. Kiryakova and F. Mainardi”, Recent history of fractional calculus," Communications in nonlinear science and numerical simulation, 16(3) 2011, pp.1140-1153.

[29]M. Meershaer and C. Tadjeran", Finite difference approximations for two-sided space fractional partial differential equations," App. Numer. Math., 56 (2006) pp.80-9

[30] R. Metzler and J. Klafter", The restaurant at the end of the random walk: recent developments in the description of anomalous transport by fractional dynamics," Journal of Physics A: Mathematical and General, 37(31) (2004) R161.

[31] S. Momani, A.A. Rqayiq and D. Baleanu", A nonstandard finite difference scheme for two-sided space-fractional partial differential equations," International Journal of Bifurcation and Chaos, 22(04) 2012, pp.1250079.

[32] S. Momani, and Z. Odibat", Numerical comparison of methods for solving linear differential equations of fractional order," Chaos Solitons Fractals., 312007 pp.1248-1255.

[33] O Nikan, M. Molavi-Arabshai, H. Jafari, "Numerical simulation of the nonlinear fractional regularized longwave model arising in ion acoustic plasma waves," Discrete and Continuous Dynamical Systems 14(10) 2021, pp.3685-3701, doi: 10.3934/dcdss.2020466.

[34]I. Podlubny, "Fractional Differential Equations," Academic Press, San Diego (1999).

[35]H. Qiao, Z.Liu and A. Cheng, "A fast compact finite difference method for fractional Cattaneo equation based on Caputo-Fabrizio derivative," Mathematical Problems in Engineering, (2020) 2020.

[36] M. Rehman and R. Ur Ali Khan, "The Legendre wavelet method for solving fractional differential equations," Comnun. Nonl. Sci. Numer. Simu., 16 2011, pp.41634173.

[37]L. Sattenspie and A. Herring, "Simulating the Effect of Quarantine on the Spread of the 1918-19 Flu in Central Canada," Bulletin of Mathematical Biology 65(1):1-26, 2003, DOI: 10.1006/bulm.2002.0317.

[38]L. Suarez and A. Shokooh, "An eigenvector expansion method for the solution of motion containing fractional derivative," J. Appl. Mech., 64 (1997) pp. 629-635.

[39]Z.Z. Sun, and X.N. Wu, "A fully discrete difference scheme for a diffusion-wave system," Appl. Numer. Math. 56 2006, pp.193-209

[40] N. Sweilam and T. Assiri, "Non-standard finite difference schemes for solving fractional order hyperbolic partial differential equations with riesz fractional derivative," Journal of Fractional Calculus and Applications 7(1) 2016, pp. 46-60.

[41] N.H. Sweilam, D.M. El-Sakout and M.M. Muttardi, "Compact finite difference method to numerically solving a stochastic fractional advection-diffusion equation," Advances in Difference Equations, 2020(1) (2020) 1-20.
[42] Y.M. Wang, "A compact finite difference method for a class of time fractional convection-diffusion-wave equations with variable coefficients," Numerical Algorithms, 70(3) 2015, pp.625-651.

[43] Q. Yang, F. Liu and I. Turner, "Numerical methods for fractional partial differential equations with Riesz space fractional derivatives," Applied Mathematical Modelling, 34(1) 2010, pp.200-218.

[44] Y. Zhang, D.A. Benson and D.M. Reeves, "Time and space nonlocalities underlying fractional-derivative models, Distinction and literature review of field applications," Advances in Water Resources, 32(4) 2009, pp.561-581.

\section{Creative Commons Attribution License 4.0 (Attribution 4.0 International, CC BY 4.0)}

This article is published under the terms of the Creative Commons Attribution License 4.0 https://creativecommons.org/licenses/by/4.0/deed.en_US 